\title{
Favorable long-term renal outcome following pediatric liver transplantation
}

\author{
Su Young Hong ${ }^{1}$, Namjoon Yi', Byung Min Yoo', Suk Kyun Hong ${ }^{1}$, Yo Han Ahn², Hee Gyung Kang ${ }^{2}$, YoungRok Choi ${ }^{1}$, \\ Kwang-Woong Lee ${ }^{1}$, Kyung-Suk Suh ${ }^{1}$ \\ ${ }^{1}$ Department of Surgery, Seoul National University College of Medicine, Seoul, Korea
${ }^{2}$ Department of Pediatrics-Nephrology, Seoul National University College of Medicine, Seoul, Korea
}

Background: Renal dysfunction is one of the critical issues of long-term outcome after liver transplantation (LT). Post-transplant renal function in adult transplant patients is well described, however, little is known about its prevalence in pediatric transplant patients.

Methods: From March 1999 to May 2016, 225 recipients underwent pediatric LT in Seoul National University Hospital. Patients with follow-up period less than 3 months or preoperative chronic kidney disease (CKD) were excluded. Cumulative incidence of CKD (defined as a glomerular filtration rate of $60 \mathrm{~mL} / \mathrm{min} / 1.73 \mathrm{~m}^{2}$ of body-surface area or less or the development of end-stage renal disease) was determined using a Kaplan-Meier method.

Results: The median age at LT was 2 years (range, 0.2-17 years). During a median follow-up of 150 months, CKD developed in nine patients (4.41\%). Of these patients, three patients underwent renal transplantation. The 1-, 5-, 10-year renal survival with CKD as the event was $99 \%, 97.9 \%$, and $96.1 \%$, respectively. In the adult group who received LT during the same period, The 1-, 5-, 10year renal survival was $96.2 \%, 85.6 \%$, and $79.4 \%$, respectively, which showed significant difference compared to pediatric group $(P<0.001)$. In a multivariate Cox regression model, hepatic artery thrombosis $(P<0.0001)$ and primary liver diseases with potential renal involvement $(P=0.033)$ were associated with $C K D$.

Conclusions: Renal function can be highly preserved following pediatric LT even in the long-term period, which is distinct finding from adult LT patients. However, more attention should be paid to patients with hepatic artery thrombosis and primary liver diseases with potential renal involvement to better improve renal outcome after pediatric LT.

Corresponding author: Namjoon Yi

E-mail: gsleenj@hanmail.net

(c) The Korean Society for Transplantation

This is an Open Access article distributed under the terms of the Creative Commons Attribution Non-Commercial License (http://creativecommons.org/licenses/by-nc/4.0/) which permits unrestricted non-commercial use, distribution, and reproduction in any medium, provided the original work is properly cited. 